\title{
Oncological feasibility of laparoscopic subtotal gastrectomy compared with laparoscopic proximal or total gastrectomy for CT1NOMO gastric cancer in the upper gastric body
}

\author{
Yosuke Kano $^{1} \cdot$ Manabu Ohashi $^{1}$ (D) Satoshi Ida ${ }^{1} \cdot$ Koshi Kumagai $^{1} \cdot$ Souya Nunobe $^{1} \cdot$ Takeshi Sano $^{1} \cdot$ Naoki Hiki $^{1}$
}

Received: 1 January 2019 / Accepted: 24 February 2019 / Published online: 4 March 2019

(c) The International Gastric Cancer Association and The Japanese Gastric Cancer Association 2019

\begin{abstract}
Background The upper gastric body is of particular interest in relation to gastrectomy because this area includes a border; that is, both distal and proximal gastrectomy for early gastric cancer can involve this area. Laparoscopic subtotal gastrectomy (LsTG) is reported to be suitable procedure compared with laparoscopic proximal and total gastrectomy (LPG, LTG), regarding postoperative nutritional status and surgical safety. However, whether LsTG is an oncologically acceptable procedure for early gastric cancer in the upper gastric body is unclear.

Methods We analyzed 215 patients with cT1N0M0 gastric cancer limitedly located in the upper gastric body. The frequency of conversion from each intended procedure to an alternative procedure, the width of the pathological margin, the incidence of lymph node metastasis at each station and the 3-year overall survival (OS) and relapse-free survival (RFS) were evaluated. Results LsTG was planned for 65 patients, and LPG for 72 and LTG for 78, respectively. Conversion to other procedures was required in about $10 \%$ of patients for whom LsTG or LPG was planned. The width of the pathological margin in patients who underwent LsTG was significantly shorter than patients who underwent the others. No patients who underwent LsTG, LPG or LTG had metastases in station no. 2 or 4sa lymph node. The 3-year OS and RFS rates of patients for whom each procedure was planned were not different.

Conclusions LsTG could be an oncologically acceptable procedure for cT1N0M0 gastric cancer in the upper gastric body. LsTG could be one option for such disease.
\end{abstract}

Keywords Early gastric cancer · Upper gastric body $\cdot$ Laparoscopic subtotal gastrectomy $\cdot$ Laparoscopic proximal gastrectomy $\cdot$ Laparoscopic total gastrectomy

\section{Introduction}

The incidence of gastric cancer in the upper-third of the stomach has been increasing in Asia during recent years [1-3]. In general, early gastric cancer (EGC) of the upperthird of the stomach is managed either by total or proximal gastrectomy, which have recently been performed

Yosuke Kano and Manabu Ohashi contributed equally to this study.

Manabu Ohashi

manabu.ohashi@jfcr.or.jp

1 Department of Gastroenterological Surgery,

Gastroenterological Center, Cancer Institute Hospital, Japanese Foundation for Cancer Research, 3-8-31 Ariake, Koto-ku, Tokyo 135-8550, Japan laparoscopically (LTG or LPG). However, there are some problems associated with these procedures, namely LTG is a very recently introduced procedure compared with laparoscopic distal gastrectomy. In particular, the technique for performing esophagojejunostomy is controversial $[4,5]$. Gastroesophageal reflux can occur after LPG, prompting various types of reconstruction, a standard procedure not yet having been established. Moreover, because the incidence of remnant stomach cancer is relatively high, ongoing postoperative surveillance of the remnant stomach is required [6, 7]. Several studies have found that proximal gastrectomy has little benefit over total gastrectomy in terms of nutrition and weight loss $[8,9]$. Considering these data, laparoscopic subtotal gastrectomy (LsTG) with a very small remnant stomach has been also performed for upper-third EGC; several studies have shown this procedure has surgical and nutritional benefits over LPG or LTG [10, 11]. 
The upper gastric body, a term that is mainly used in endoscopic examination, is defined as the upper-third of the region from the cardia to the angle, which is included in the upper-third of the stomach. The upper gastric body is of particular interest in relation to gastrectomy for EGC because this area includes a border; that is, both distal and proximal gastrectomy for ECG can involve this area. However, the area of lymph node dissection in distal gastrectomy differs from that in proximal gastrectomy. According to the Japanese Classification of Gastric Carcinoma (JCGC), 13th edition [12], optimal lymph node dissection of the upperthird of the stomach includes stations no. 2 and 4 sa. Therefore, proximal or total gastrectomy should be performed for EGC in the upper gastric body. However, according to the current Japanese Gastric Cancer Treatment Guidelines (JGCTG) (4th edition), the extent of lymph node dissection is determined by the type of gastrectomy rather than the tumor location [13]. Thus, according to the JGCTG, LPG, LsTG and LTG can be performed for EGC in the upper gastric body even though LPG and LsTG are very different types of gastrectomy.

Several studies have reported that LsTG is suitable for ECG in the upper-third of the stomach as far as safety and nutritional aspects are concerned [10, 11]. However, oncological problems to be considered with LsTG include a possible insufficient proximal margin because of the proximity to the cardia and risks of inadequate lymph node dissection, especially failure to dissect stations no. 2 and 4a. Thus, whether LsTG is an oncologically acceptable procedure for ECG in the upper gastric body is thus far unclear; no studies have specifically investigated these points.

In the present study, surgical outcomes of patients who had undergone laparoscopic gastrectomy for cT1N0M0 gastric cancer in the upper gastric body were retrospectively analyzed from an oncological perspective. The information obtained from this study will help in determining whether LsTG is an oncologically acceptable procedure for cT1N0M0 in the upper gastric body. To the best of our knowledge, this is the first report to focus on EGC located in the upper gastric body and to compare the oncological outcomes of LsTG with those of LTG and LPG.

\section{Patients and methods}

\section{Patients}

The data of patients who had undergone R0 gastrectomy in the Cancer Institute Hospital, Tokyo, Japan, between January 2006 and December 2016 were retrospectively extracted from our prospectively input database and reviewed. A total of 215 patients for whom laparoscopic gastrectomy was planned for clinically diagnosed cT1NOM0 (cstage I) gastric cancer located only in the upper gastric body were eligible. The upper gastric body was defined as the upper-third of the gastric body, which is from the subcardial area to the angle (excluding the esophagogastric junction according to the Nishi classification) (Fig. 1). Tumor location and gastric circumference were ascertained by gastroscopy and clinical depth of tumor by endoscopy, upper gastrointestinal series, and computed tomography findings. Endoscopic ultrasonography was performed in some cases. Exclusion criteria were as follows: double cancer involving the stomach and another organ, receiving neoadjuvant chemotherapy, conversion to laparotomy because of intra-abdominal findings such as peritoneal dissemination, tumor depth suspected to be more than $\mathrm{T} 1 \mathrm{~b}$, and severe adhesions, and tumor extending to fundus, esophagogastric junction (EGJ), or middle gastric body. Clinical stage was classified according to the JCGC (14th edition) [14]. Differentiated types included papillary and tubular adenocarcinomas; undifferentiated types included poorly differentiated adenocarcinoma, signet ring cell carcinoma, and mucinous adenocarcinoma. This study was approved by the Institutional Review Board of the Cancer Institute Hospital.

\section{Surgical procedure for LsTG (Fig. 2)}

LsTG was defined as laparoscopic distal gastrectomy for tumor located in the upper-third of the stomach or tumor invading to the area. The basic indications for LsTG were as follows: (1) EGC diagnosed as cT1NOM0; and (2) more than $3 \mathrm{~cm}$ from the proximal boundary of the tumor to the EGJ according to endoscopic findings. The final decision whether LsTG could be performed was made based on a virtual transected line of the stomach, which was determined according to proximal markings put on places where biopsied specimens were pathologically negative for cancer. If

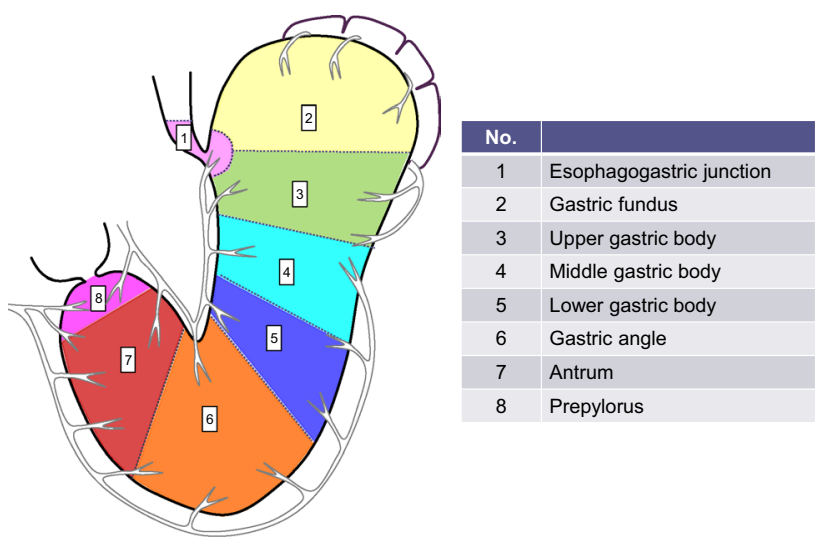

Fig. 1 Diagram showing classification of sections of the stomach. The upper gastric body (3) is defined as the upper-third of the region from the cardia to the angle and is included in the upper-third of the stomach 


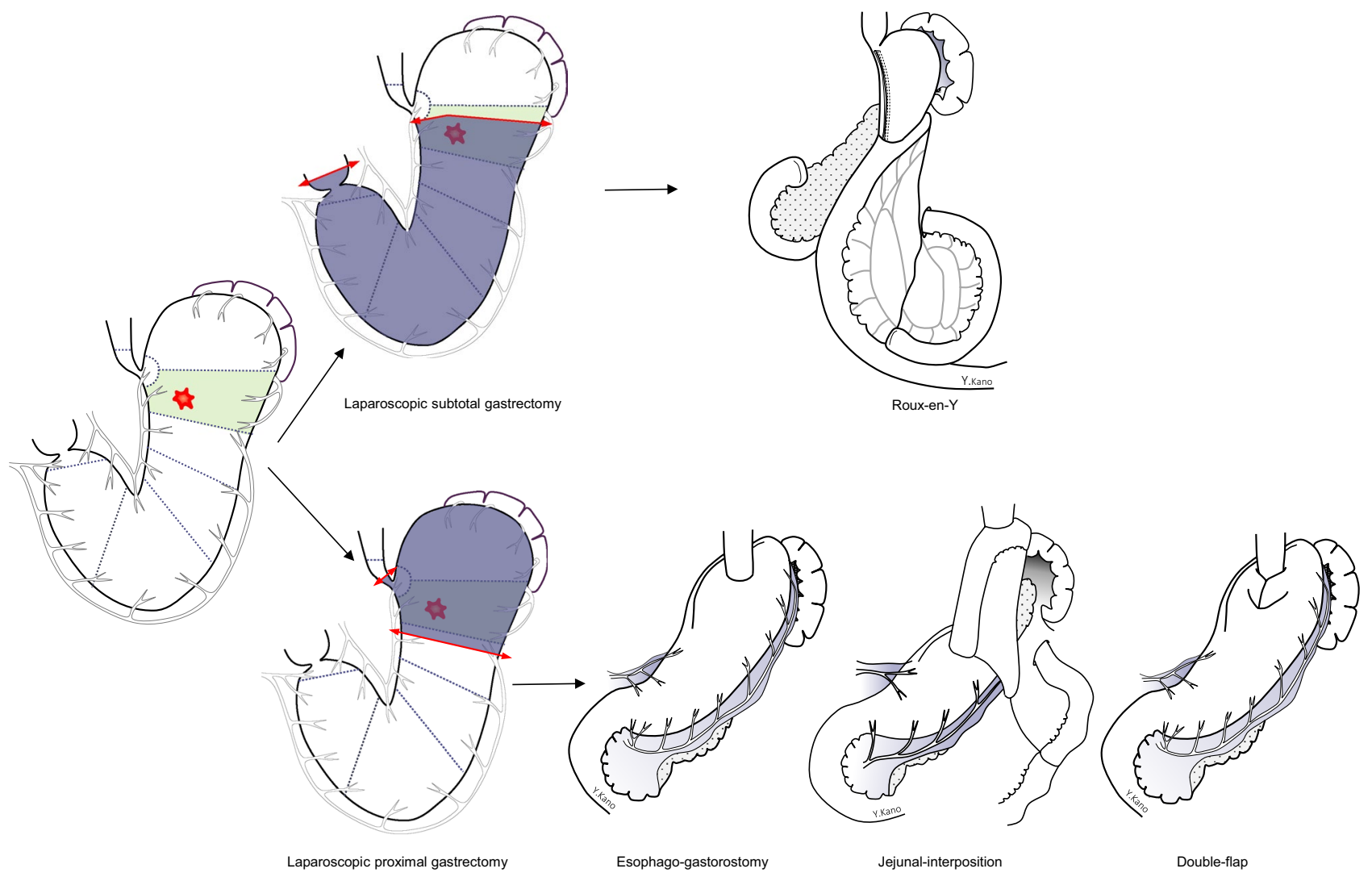

Fig. 2 Schema showing resection and reconstruction in laparoscopic subtotal gastrectomy and laparoscopic proximal gastrectomy. In laparoscopic subtotal gastrectomy, a Roux-en-Y type is constructed, whereas in laparoscopic proximal gastrectomy, one of three types of

LsTG was contraindicated, LPG or LTG were considered; that is, LsTG was the procedure of choice. Selection of LPG or LTG depended on the era; namely, in the earlier part of the study period, LsTG and LTG were preferred, whereas LPG with a double-flap technique (DFT) [15] has been performed more often recently.

LsTG included modified D2 lymphadenectomy (D1+ no. $8 \mathrm{a}, 9$, and $11 \mathrm{p}$ ). A detailed description of this surgical procedure of LsTG has previously been reported by our team $[16,17]$. More recently, the following modifications to the LsTG procedure were introduced. The stomach was transected under the guidance of intraoperative endoscopy using an endoscopic linear stapler [18]. The gastric transection line was usually determined according to marking clips; however, recently small, fine, flat cautery marks have been placed on the pathologically intact mucosa proximal to the tumor boundary for tumors less than $3 \mathrm{~cm}$ from the cardia and/or fornix [19]. After transection of the stomach, the cut edge of the proximal margin was immediately submitted for frozen section analysis. If the edge was positive for cancer, a complete gastrectomy was performed in the same surgery. When reconstructing by gastrojejunostomy, reconstruction is used, namely esophagogastrostomy, jejunal interposition or double-flap technique. Each procedure was selected according to the era in the study period according to current policies

a 25-mm circular stapler (Orvil Covidien, Mansfield, MA, USA) was usually used; however, an endoscopic linear stapler has recently been used.

\section{Surgical procedure for LPG (Fig. 2)}

In LPG, D1+ lymphadenectomy was also performed. Marking clips were placed on the pathologically intact mucosa distal to the tumor boundary. The stomach was transected according to marks confirmed by manual handling or intraoperative endoscopic observation. When reconstructing after LPG, an esophagogastric anastomosis, jejunal interposition (JI), or DFT was constructed according to the policy in the relevant era of the study period. When creating an esophagogastric anastomosis, which was adopted during the earliest era, a 25-mm circular stapler was used and an esophagogastrostomy made on the anterior wall of the remnant stomach. In JI, the proximal jejunum was brought up for anastomosis with the esophagus and remnant stomach. An esophagojejunostomy was constructed in an end-to-side fashion using a circular stapler, and a jejunogastrostomy was created on the anterior wall of the remnant stomach by using a linear 
stapler. In DFT, which was performed during the most recent era, most procedures were based on Kamikawa's open surgical methods [15]. An esophagogastrostomy was constructed on the anterior wall in a layer-to-layer fashion by hand stitching, after which hinged flaps made of the seromuscular layer of the stomach were used to cover the anastomosis.

\section{Surgical procedure for LTG}

In LTG, D1+ lymphadenectomy was performed. A Roux-en$\mathrm{Y}$ was constructed via an antecolic route. An esophagojejunostomy was constructed using a linear stapler or a $25-\mathrm{mm}$ circular stapler. A jejunojejunostomy was made approximately $45 \mathrm{~cm}$ distal to the esophagojejunostomy in a sideto-side fashion.

\section{Evaluation and statistical analysis}

Differences in clinicopathological characteristics of patients for whom each procedure was planned were evaluated. Fisher's exact and Kruskal-Wallis tests were used for statistical analysis. The frequency of conversion from each intended procedure to an alternative procedure was calculated and the reasons for conversion were identified. The width of the pathological margin was assessed by the proximal margin in LsTG and LTG and by the distal margin in LPG. Postoperative complications were evaluated according to the Clavien-Dindo classification (C-D) [20, 21]. C-D grade 2 or higher within 30 days after surgery were regarded as an event. Fisher's exact and Kruskal-Wallis tests were used for statistical analysis. Overall survival (OS) and relapsefree survival (RFS) were estimated using the Kaplan-Meier method and compared by the log-rank test. $P$ values less than 0.05 on all statistical tests was considered to denote statistical significance. The probability of LN metastasis was estimated with 95\% confidence intervals (95\% CI), based on the exact binominal distribution. All statistical analyses were performed with SPSS ver. 24.0 for windows (IBM, Chicago, IL, USA).

\section{Results}

\section{Patient characteristics}

The clinicopathological features of the 215 patients according to planned procedure are shown in Table 1. LsTG was planned for 65 patients, LPG for 72, and LTG for 78, respectively. There were significant differences in sex, operative time, blood loss, and duration of hospitalization after surgery. No differences were found for the other factors. Although 3 patients included in this study had pathologically serosal invasion (T4a), their serosal invasions could not be identified clinically.

\section{Oncological outcomes}

\section{Conversion from planned to an alternative procedure}

Table 2 shows oncological outcomes. Conversion to other procedures was required in about $10 \%$ of patients for whom LsTG or LPG was planned. Seven patients for whom LsTG was planned actually underwent total gastrectomy. The reasons were pathologically positive margin in 3 patients, intraoperative findings of contraindication in 3 patients, and others in 2 patients. The others contained grossly short proximal margin of the specimen and pathologically positive lymph node metastasis at the station no. $11 \mathrm{p}$, which was found intraoperatively. Six of seven patients for whom LPG was planned required conversion to total gastrectomy and one to LsTG. The main reason for conversion in four of these patients was too small a distal remnant stomach as determined by intraoperative re-assessment, whereas the main reason in the other three was a pathological positive margin.

The width of the pathological proximal margin, excluding positive margin in the LsTG specimens, was significantly smaller than that of the distal margin in the LPG specimens $(P<0.001)$, and of the proximal margin in the LTG specimens $(P<0.001)$.

\section{Incidence of lymph node metastasis}

Table 3 shows the incidence of lymph node metastasis in each lymph node station. The most frequent station harboring lymph node metastasis was no. 3 (4.7\%), the incidence of metastasis being less than $1 \%$ in the other stations. There were no lymph node metastases in stations no. 4sa, 5, 6, $10,11 \mathrm{~d}$, or 12a. One patient with pT4aN2, who required conversion from LPG to open total gastrectomy because of a positive anal margin, had no. 2 lymph node metastasis.

\section{Survival}

Figure 3 shows Kaplan-Meier estimates of OS and RFS stratified by the intended procedure. The 3-year OS rates of patients for whom LsTG, LPG, and LTG were planned were $98.4 \%, 98.6 \%$ and $97.4 \%$, respectively $(P=0.332)$. The 3 -year RFS rates of patients for whom LsTG, LPG and LTG were planned were $98.4 \%, 97.0 \%$ and $97.4 \%$, respectively $(P=0.358)$. Relapse occurred in three patients $(1.4 \%)$, two of whom had pathologically advanced disease and one had pathologically early disease.

Liver metastasis or lymphangiosis carcinomatosa occurred in two patients in the LPG group, and peritoneal 
Table 1 Patients characteristics

\begin{tabular}{|c|c|c|c|c|}
\hline & \multicolumn{3}{|c|}{ Intended procedure } & \multirow[t]{2}{*}{$P$ value } \\
\hline & LsTG $(n=65)$ & LPG $(n=72)$ & LTG $(n=78)$ & \\
\hline Sex, $n(\%)$ & & & & 0.023 \\
\hline Male & $49(75.4)$ & $47(65.3)$ & $66(84.6)$ & \\
\hline Female & $16(24.6)$ & $25(34.7)$ & $12(15.4)$ & \\
\hline Age $^{\mathrm{a}}$, years & $66(39-82)$ & $67(30-88)$ & $66(41-84)$ & 0.580 \\
\hline After ESD, $n(\%)$ & $14(21.9)$ & $21(29.2)$ & $16(20.8)$ & 0.448 \\
\hline Gastric circumference & & & & 0.448 \\
\hline Less & $13(20.3)$ & $17(23.8)$ & $18(23.4)$ & \\
\hline Ant & $6(9.4)$ & $13(18.1)$ & $6(7.8)$ & \\
\hline Gre & $12(18.8)$ & $13(18.1)$ & $14(18.2)$ & \\
\hline Post & $33(51.6)$ & $29(40.3)$ & $39(50.6)$ & \\
\hline Tumor size ${ }^{\mathrm{a}}$, mm & $27(3-60)$ & $28(2-125)$ & $30(1-75)$ & 0.147 \\
\hline Histological type, $n(\%)$ & & & & 0.119 \\
\hline Differentiated & $30(46.9)$ & $46(63.9)$ & $41(52.6)$ & \\
\hline Undifferentiated & $34(53.1)$ & $26(36.1)$ & $37(47.4)$ & \\
\hline $\mathrm{pT}, n(\%)$ & & & & 0.663 \\
\hline $\mathrm{T} 1$ & $57(89.6)$ & $64(88.9)$ & $66(85.7)$ & \\
\hline $\mathrm{T} 2$ & $4(6.0)$ & $3(4.2)$ & $8(10.4)$ & \\
\hline T3 & $2(3.0)$ & $3(4.2)$ & $3(3.9)$ & \\
\hline $\mathrm{T} 4$ & $1(1.5)$ & $2(2.8)$ & & \\
\hline $\mathrm{pN}, n(\%)$ & & & & 0.669 \\
\hline N0 & $58(90.6)$ & $66(91.7)$ & $74(96.1)$ & \\
\hline N1 & $5(7.8)$ & $4(5.6)$ & $2(2.6)$ & \\
\hline $\mathrm{N} 2$ & $1(1.6)$ & $2(2.8)$ & $1(1.3)$ & \\
\hline pStage, $n(\%)$ & & & & 0.684 \\
\hline IA & $51(79.7)$ & $61(84.7)$ & $63(81.8)$ & \\
\hline IB & $9(14.1)$ & $6(8.3)$ & $11(14.3)$ & \\
\hline IIA & $3(4.7)$ & $1(1.4)$ & $2(2.6)$ & \\
\hline IIB & $1(1.6)$ & $2(2.8)$ & & \\
\hline IIIA & & $1(1.4)$ & $1(1.3)$ & \\
\hline IIIB & & $1(1.4)$ & & \\
\hline Operative time $^{\mathrm{a}}$, min & $290(108-470)$ & $390(140-639)$ & $304(197-516)$ & $<0.001$ \\
\hline Blood loss ${ }^{\mathrm{a}}, \mathrm{mL}$ & $30(5-278)$ & $50(5-280)$ & $70(10-680)$ & $<0.001$ \\
\hline $\begin{array}{l}\text { Postoperative morbidity } \\
\text { C-D grade } \geq \text { II, } n(\%)\end{array}$ & $14(21.5)$ & $19(26.4)$ & $22(28.2)$ & 0.657 \\
\hline Hospitalization $^{\mathrm{a}}$, days & $10(8-44)$ & $12(7-45)$ & $13(8-61)$ & $<0.001$ \\
\hline
\end{tabular}

Ant anterior wall, $C-D$ Clavien-Dindo classification, ESD endoscopic submucosal dissection, Less lesser curvature, Gre greater curvature, $L P G$ laparoscopic proximal gastrectomy, $L s T G$ laparoscopic subtotal gastrectomy with a small remnant stomach, $L T G$ laparoscopic total gastrectomy, Post posterior wall

${ }^{a}$ Values are presented as median (range)

metastasis occurred in one in the LTG group. No relapses were identified in the LsTG group.

\section{Discussion}

In this study, we identified three new pieces of information concerning oncological outcomes of LsTG for cT1N0M0 gastric cancer in the upper gastric body. First, the incidence of conversion to total gastrectomy in patients for whom LsTG or LPG is planned is about $10 \%$ and the main reason for conversion is positive proximal or distal margins. In our cohort, the pathological width of the surgical margin was smaller after LsTG than after LsTG, LPG, or LTG. Second, the incidence of lymph node metastasis was quite low. At only $5 \%$, metastasis to station no. 3 was the most frequent; metastasis to other stations occurred in less than $1 \%$ of patients. There were no relapses to station no. 2 or 4 sa lymph 
Table 2 Oncological outcomes according to intended procedure

\begin{tabular}{lccc}
\hline \multicolumn{4}{c}{ Intended procedure } \\
\cline { 2 - 4 } & LsTG $(n=65)$ & LPG $(n=72)$ & LTG $(n=78)$ \\
\hline Performed procedure, $n(\%)$ & $58(89.2)$ & $1(1.4)$ & $3(3.8)$ \\
LsTG & $0(0)$ & $65(90.2)$ & $0(0)$ \\
LPG & $2(3.1)$ & $3(4.2)$ & $75(96.2)$ \\
LTG & $5(7.7)$ & $3(4.2)$ & $0(0)$ \\
OTG & $7(10.7)$ & $7(9.8)$ & $3(3.8)$ \\
Conversion to other gastrectomy, $n(\%)$ & & & $0(0)$ \\
Reason for conversion, $n(\%)$ & $3(4.6)$ & $3(4.2)$ & $3(3.8)$ \\
Positive margin & $2(3.1)$ & $4(5.6)$ & $0(0)$ \\
Intraoperative findings & $2(3.1)$ & $0(0)$ & $35^{\mathrm{b}}(10-90)$ \\
Others & $15^{\mathrm{b}}(0-35)$ & $25^{\mathrm{b}}(7-55)$ & \\
Pathological margin ${ }^{\mathrm{a}}$, mm (range) & & & \\
\hline
\end{tabular}

$L P G$ laparoscopic proximal gastrectomy, $L s T G$ laparoscopic subtotal gastrectomy with a small remnant stomach, $L T G$ laparoscopic total gastrectomy, $O T G$ open total gastrectomy

${ }^{a}$ Excluding patients converted to other type of gastrectomy

${ }^{\mathrm{b}}$ Significant difference between LsTG versus LPG $(P<0.001)$ and LsTG versus LTG $(P<0.001)$

\begin{tabular}{llllr}
\hline & $\begin{array}{l}\text { No. of patients with LN } \\
\text { dissection }\end{array}$ & $\begin{array}{l}\text { No. of patients with LN } \\
\text { metastasis }\end{array}$ & $\begin{array}{l}\text { Incidence of LN } \\
\text { metastasis (\%) }\end{array}$ & 95\% CI (\%) \\
\hline No. 1 & 215 & 1 & 0.5 & $0-2.5$ \\
No. 2 & 159 & 1 & 0.6 & $0-3.5$ \\
No. 3 & 215 & 10 & 4.7 & $2.3-8.4$ \\
No. $4 \mathrm{sa}$ & 166 & 0 & 0 & $0-1.8$ \\
No. $4 \mathrm{sb}$ & 215 & 1 & 0.5 & $0-2.5$ \\
No. $4 \mathrm{~d}$ & 164 & 1 & 0.6 & $0-3.4$ \\
No. 5 & 150 & 0 & 0 & $0-2.0$ \\
No. 6 & 150 & 0 & 0 & $0-2.0$ \\
No. 7 & 215 & 2 & 0.9 & $0.1-3.3$ \\
No. $8 \mathrm{a}$ & 215 & 2 & 0.9 & $0.1-3.3$ \\
No. 9 & 215 & 1 & 0.5 & $0-2.5$ \\
No. 10 & 19 & 0 & 0 & $0-14.6$ \\
No. $11 \mathrm{p}$ & 215 & 2 & 0.9 & $0.1-3.3$ \\
No. $11 \mathrm{~d}$ & 36 & 0 & 0 & $0-8.0$ \\
No. $12 \mathrm{a}$ & 10 & 0 & 0 & $0-25.9$ \\
\hline
\end{tabular}

CI confidence interval, $L N$ lymph node

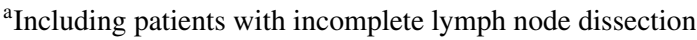

Table 3 Incidence of lymph node metastasis in each lymph node station $(n=215)$ nodes. Third, the OS and RFS of patients for whom LsTG was planned were the same as those of patients for whom LPG or LTG were planned. No patients who had undergone LsTG developed recurrences. These new findings show that LsTG for cT1N0M0 gastric cancer at the upper gastric body is oncologically acceptable, ensuring an adequate surgical margin and with an incidence of lymph node metastasis and survival comparable to those of LPG and LTG.

It is more difficult to perform limited gastrectomy for cT1N0M0 gastric cancer in the upper gastric body; as evidenced by patients with lesions located in other areas not requiring conversion to other types of gastrectomy as frequently. The major reason for conversion was positive surgical margin. It seems that it is easier to adjust the surgical margin, when performing LPG, because the esophagus is transected for the proximal margin and the middle gastric body for the distal margin. However, the stomach is transected as close to the distal boundary of the tumor as possible when performing LPG because the aim is to preserve as large a remnant stomach as possible to optimize function. In contrast, it is technically difficult to ensure the proximal margin when performing LsTG because the stomach must be 

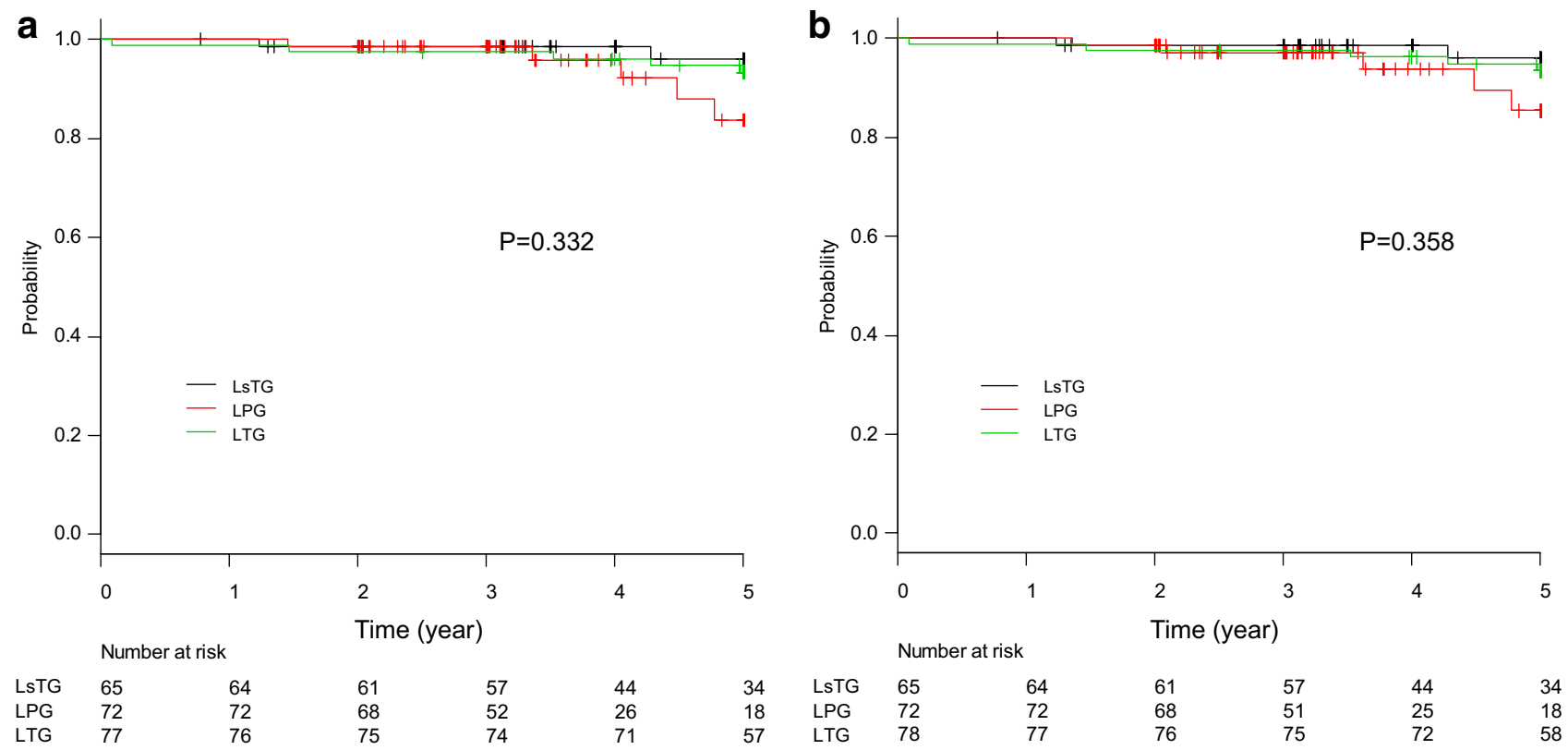

Fig. 3 Kaplan-Meier estimates of overall survival (a) and recurrence-free survival (b) by planned procedures. No differences were found among the procedures in either overall or recurrence-free survival

transected between the cardia and the boundary of the lesion, which is very narrow. However, the incidence of positive margin was similar for these procedures in this study, albeit higher than that of other locations of the stomach [22, 23]. Precise preoperative diagnosis and collaboration between endoscopic and laparoscopic techniques when performing gastric transection are likely the main reasons for his difference. We use narrow-band imaging and magnifying endoscopy to determine the precise tumor border and take biopsies from the mucosa proximal or distal to the boundary (negative biopsies) to help us to determine the optimal transection line through the stomach. Furthermore, markings based on the results of negative biopsies and use of intraoperative endoscopy enable precise gastric transection under laparoscopic view [18, 19], patients whose proximal or distal surgical margin was positive for cancer even after implementation of these modalities were found to have unexpected tumor spread below the submucosal or deeper layer. Although unexpected tumor spread can be found at any location in the stomach, a high incidence of positive margin because of the narrow region for transecting the stomach is particular to limited gastrectomy at the upper gastric body.

More than half the patients who underwent LsTG in this study had pathological proximal margins of less than $1.5 \mathrm{~cm}$; however, no patients developed local recurrence at the cut edge of the remnant stomach. Kim et al. have proposed that a $>1 \mathrm{~mm}$ pathological margin is adequate in gastrectomy for pathologically confirmed EGC [24]. However, LsTG is performed on the basis of clinical findings. Thus, intraoperative frozen section assessment of the surgical margin is indispensable in this procedure. If the surgical margin is positive for cancer or the depth of the tumor may be deeper than the submucosal layer, there should be no hesitation about intraoperative conversion to complete gastrectomy.

LsTG includes adequate lymph node dissection for cT1N0M0 gastric cancer in the upper gastric body. Because the upper gastric body is a very small area of the stomach, the incidence of lymph node metastasis in all stations from cT1N0M0 gastric cancer was quite low and occurred only in patients undergoing LPG or LTG, one of whom had metastasis in station no. 2. This patient had pT4aN2 disease and underwent conversion to total gastrectomy because of intraoperative findings of serosal invasion and lymph node metastasis. Thus, no patients with cT1 disease in this study, including those in whom it was diagnosed intraoperatively, had metastases in station no. 2 or 4 sa lymph nodes, which implies that there are no oncological reasons to dissect stations no. 2 and 4sa lymph nodes, provided LsTG for cT1N0M0 in the upper gastric body is technically possible. Considering that many metastases were found in stations no. $3,7,9$ or $11 \mathrm{p}$ in this study, the most important lymphatic route to dissect for cT1N0M0 gastric cancer is along the left gastric artery at the upper gastric body. Because stations no. 5 and 6 did not contain metastases, total gastrectomy to dissect lymph nodes around the pylorus is not necessary.

Types of gastrectomy did not influence survival outcomes of patients with cT1N0M0 gastric cancer in the upper gastric body who underwent curative surgery. The only three relapses were all distant metastases, indicating that not only LsTG, but also LPG and LTG, are 
oncologically acceptable. The oncological feasibility of these procedures contains the feasibility of laparoscopic gastrectomy and that of subtotal, proximal or total gastrectomy for cT1N0M0 gastric cancer in the upper gastric body. Recently, a pivotal phase III clinical trial in Korea: the KLASS-01 demonstrated the oncological feasibility of laparoscopic distal gastrectomy for stage I gastric cancer [25]. As the KLASS-01 result can be extrapolated to other types of laparoscopic gastrectomies, the favorable survival outcomes in our study may present the oncological feasibility of subtotal, proximal or total gastrectomy for cT1N0M0 gastric cancer in the upper gastric body. According to these considerations, the optimal procedure for patients with cT1N0M0 gastric cancer in the upper gastric body may depend on postoperative outcomes other than survival, such as symptoms, body weight, anemia, laboratory data and so on.

This study had several limitations. First, it was a retrospective single-institution study. Although the patient cohort was relatively larger, each group was small when the patients were grouped according to the three types of gastrectomy. Additionally, although the incidence of lymph node dissection would ideally be calculated for LTG, we calculated only an overall figure because so few patients underwent LTG. Second, preoperative selection of procedures took many factors into consideration, including location of the tumor, patients' age, performance status, surgeons' preference, treatment period, trends in treatment and so on. Although we only included patients with cT1N0M0 gastric cancer located in the upper gastric body, each patient and tumor would have been slightly different, which may make it difficult to generalize our findings..

In conclusion, LsTG could be an oncologically acceptable procedure for cT1N0M0 gastric cancer in the upper gastric body, as are LPG and LTG. LsTG could be one option for such disease. To determine which procedure is optimal for patients with cT1N0M0 gastric cancer in the upper gastric body, outcomes other than oncological ones should be investigated and compared between procedures.

Acknowledgements The authors declare that no external funding was received for this study.

\section{Compliance with ethical standards}

Conflict of interest The authors have no conflicts of interest to declare.

Human rights statement All procedures followed were in accordance with the ethical standards of the institutional and national committees governing human experimentation and in compliance with the Helsinki Declaration of 1964 and later versions.

Informed consent Informed consent or an appropriate substitute was obtained from all patients prior to their inclusion in the study.

\section{References}

1. Deans C, Yeo MS, Soe MY, Shabbir A, Ti TK, So JB. Cancer of the gastric cardia is rising in incidence in an Asian population and is associated with adverse outcome. World J Surg. 2011;35:617-24.

2. Zhou Y, Zhang Z, Zhang Z, Wu J, Ren D, Yan X, et al. A rising trend of gastric cardia cancer in Gansu Province of China. Cancer Lett. 2008;269:18-25.

3. Ahn HS, Lee HJ, Yoo MW, Jeong SH, Park DJ, Kim HH, et al. Changes in clinicopathological features and survival after gastrectomy for gastric cancer over a 20 -year period. Br J Surg. 2011;98:255-60.

4. Gong CS, Kim BS, Kim HS. Comparison of totally laparoscopic total gastrectomy using an endoscopic linear stapler with laparoscopic-assisted total gastrectomy using a circular stapler in patients with gastric cancer: a single-center experience. World J Gastroenterol. 2017;23:8553-61.

5. Lu X, Hu Y, Liu H, Mou T, Deng Z, Wang D, et al. Short-term outcomes of intracorporeal esophagojejunostomy using the transorally inserted anvil versus extracorporeal circular anastomosis during laparoscopic total gastrectomy for gastric cancer: a propensity score matching analysis. J Surg Res. 2016;200:435-43.

6. Iwata Y, Ito S, Misawa K, Ito Y, Komori K, Abe T, et al. Incidence and treatment of metachronous gastric cancer after proximal gastrectomy. Surg Today. 2018;48:552-7.

7. Nozaki I, Kurita A, Nasu J, Kubo Y, Aogi K, Tanada M, et al. Higher incidence of gastric remnant cancer after proximal than distal gastrectomy. Hepatogastroenterology. 2007;54:1604-8.

8. An JY, Youn HG, Choi MG, Noh JH, Sohn TS, Kim S. The difficult choice between total and proximal gastrectomy in proximal early gastric cancer. Am J Surg. 2008;196:587-91.

9. Chen S, Li J, Liu H, Zeng J, Yang G, Wang J, et al. Esophagogastrostomy plus gastrojejunostomy: a novel reconstruction procedure after curative resection for proximal gastric cancer. J Gastrointest Surg. 2014;18:497-504.

10. Furukawa H, Kurokawa Y, Takiguchi S, Tanaka K, Miyazaki Y, Makino T, et al. Short-term outcomes and nutritional status after laparoscopic subtotal gastrectomy with a very small remnant stomach for cStage I proximal gastric carcinoma. Gastric Cancer. 2018;21:500-7.

11. Nakauchi M, Suda K, Nakamura K, Shibasaki S, Kikuchi K, Nakamura T, Uyama I, et al. Laparoscopic subtotal gastrectomy for advanced gastric cancer: technical aspects and surgical, nutritional and oncological outcomes. Surg Endosc. 2017;31:4631-40.

12. Japanese Gastric Cancer Association. Japanese classification of gastric carcinoma-2nd English edition. Gastric Cancer. 1998;1:10-24.

13. Japanese Gastric Cancer Association. Japanese gastric cancer treatment guidelines 2014 (ver. 4). Gastric Cancer. 2017;20:1-19.

14. Japanese Gastric Cancer Association. Japanese classification of gastric carcinoma: 3rd English edition. Gastric Cancer. 2011;14:101-12.

15. Kamikawa Y, Kobayashi T, Kamiyama S, Satomoto K. A new procedure of esophagogastrostomy to prevent reflux following proximal gastrectomy (in Japanese). Shoukakigeka. 2001;24:1053-60.

16. Hiki N, Nunobe S, Kubota T, Jiang X. Function-preserving gastrectomy for early gastric cancer. Ann Surg Oncol. 2013;20:2683-92.

17. Jiang X, Hiki N, Nunobe S, Nohara K, Kumagai K, Sano T, et al. Laparoscopy-assisted subtotal gastrectomy with very small remnant stomach: a novel surgical procedure for selected early gastric cancer in the upper stomach. Gastric Cancer. 2011;14:194-9.

18. Kawakatsu S, Ohashi M, Hiki N, Nunobe S, Nagino M, Sano T. Use of endoscopy to determine the resection 
margin during laparoscopic gastrectomy for cancer. Br J Surg. 2017; 104:1829-36.

19. Kamiya S, Ohashi M, Ida S, Kumagai K, Nunobe S, Sano T, et al. Laparoscopic subtotal gastrectomy with a new marking technique, endoscopic cautery marking: preservation of the stomach in patients with upper early gastric cancer. Surg Endosc. https:// doi.org/10.1007/s00464-018-6272-3 (Online Jun 19, 2018).

20. Dindo D, Demartines N, Clavien PA. Classification of surgical complications: a new proposal with evaluation in a cohort of 6336 patients and results of a survey. Ann Surg. 2004;240:205-13.

21. Clavien PA, Barkun J, de Oliveira ML, Vauthey JN, Dindo D, Schulick RD, et al. The Clavien-Dindo classification of surgical complications: five-year experience. Ann Surg. 2009;250:187-96.

22. Sun Z, Li DM, Wang ZN, Huang BJ, Xu Y, Li K, Xu HM. Prognostic significance of microscopic positive margins for gastric cancer patients with potentially curative resection. Ann Surg Oncol. 2009;16:3028-37.

23. Cho BC, Jeung HC, Choi HJ, Rha SY, Hyung WJ, Cheong JH, Noh SH, Chung HC. Prognostic impact of resection margin involvement after extended (D2/D3) gastrectomy for advanced gastric cancer: a 15-year experience at a single institute. J Surg Oncol. 2007;95:461-8.

24. Kim BS, Oh ST, Yook JH, Kim HS, Lee IS, Kim BS. Appropriate gastrectomy resection margins for early gastric carcinoma. J Surg Oncol. 2014;109:198-201.

25. Kim HH, Han SU, Kim MC, Kim W, Lee HJ, Ryu SW, et al., Korean Laparoendoscopic Gastrointestinal Surgery Study (KLASS) Group. Effect of laparoscopic distal gastrectomy vs open distal gastrectomy on long-term survival among patients with stage I gastric cancer: the KLASS-01 randomized clinical trial. JAMA Oncol. https://doi.org/10.1001/jamaoncol.2018.6727. (Online Feb 7, 2019).

Publisher's Note Springer Nature remains neutral with regard to jurisdictional claims in published maps and institutional affiliations. 